\section{The blubbery protrusions: Lipoma arborescens}

\author{
Anubha Bajaj* \\ Histopathologist, AB. Diagnostics, New Delhi, India
}

\section{Preface}

Lipoma arborescens is an exceptional condition comprised of frond-like excrescences of mature adipose tissue. The condition was originally described by Albert Hoffa in 1904 with characteristic morphology of macroscopic, villous, frond- like excrescences recapitulating a tree-like appearance, as denominated by the term "arborescens" [1]. Lipoma arborescens is additionally designated as "diffuse articular lipomatosis", "villous lipomatous proliferation of synovial membrane" or "diffuse lipoma of joint". The essentially benign condition appears within large joints and typically exhibits adipose tissue infiltration of sub-synovial connective tissue. Synovial sheaths of tendons are infrequently incriminated $[1,2]$.

\section{Disease characteristics}

Of obscure aetiology, lipoma arborescens is posited to be a nonspecific, reactive proliferation of synovial adipose tissue secondary to chronic trauma or inflammatory stimulus, in contrast to an emergent neoplastic process. The condition preponderantly implicates synovial layer of joints and bursae and represents beneath $<1 \%$ of mature adipose tissue neoplasms [2,3]. The exceptional condition arises in elderly population, typically within fifth to seventh decade although young individuals can be implicated. Nevertheless, lipoma arborescens is observed between 9 years to 68 years. Gender prevalence of the disorder is equivalent [2,3]. Generally, lesions are sporadic. However, the condition is associated with traumatic joint injury, degenerative joint disease, collagen vascular disorders, chronic arthritis or osteoarthritis $[2,3]$.

\section{Clinical elucidation}

Common clinical symptoms are joint swelling with frequent joint effusion and an arthralgia of varying duration. Usually an insidious disease onset with occurrence of a persistent, painless swelling of incriminated joint of an extended duration followed by progressive pain associated with sporadic episodes of joint effusion is exemplified. Additionally, discontinuous, exacerbating pain and joint swelling is discerned, contingent

\author{
More Information \\ *Address for Correspondence: Anubha Baja, \\ Histopathologist, AB. Diagnostics, New Delhi, \\ India, Tel: 00911141446785; \\ Email: anubha.bajaj@gmail.com \\ Submitted: October 29, 2020 \\ Approved: December 14, 2020 \\ Published: December 15, 2020 \\ How to cite this article: Bajaj $A$. The blubbery \\ protrusions: Lipoma arborescens. Ann Dermatol \\ Res. 2020; 4: 009-013. \\ DOI: 10.29328/journal.adr.1001011 \\ Copyright: @ 2020 Bajaj A. This is an open \\ access article distributed under the Creative \\ Commons Attribution License, which permits \\ unrestricted use, distribution, and reproduction \\ in any medium, provided the original work is \\ properly cited. \\ (D) Check for updates \\ OPEn Access
}

to entrapment of hypertrophied, adipose tissue -rich villi between mobile joint surfaces [2,3]. Knee joint is frequently and unilaterally incriminated, particularly suprapatellar bursa. Bilateral knee joints are implicated in an estimated $20 \%$ subjects. Also, lipoma arborescens is observed within diverse joints such as the shoulder, hip, elbow, ankle, wrist along with periarticular bursae and tendon sheaths. Associated joints or tendon sheaths are infrequently involved [3,4]. Although commonly mono-articular, bilateral or poly-articular lesions are observed in secondary subtype of lipoma arborescens. Of dual aetiology, lipoma arborescens appears due to "primary" and "secondary" factors contingent to age of disease onset and concomitant, precipitating conditions $[3,4]$.

The exceptional, idiopathic, primary subcategory of lipoma arborescens can arise within extra-articular tissues of synovial origin such as bursae or tendon sheaths and are discerned in pertinent joints as the sub-acromial bursa of shoulder, bicipito-radial bursa, ankle and radiocarpal periarticular tendon sheaths. Primary subtype emerges within younger subjects, commonly between second and third decade. Biochemical parameters such as erythrocyte sedimentation rate (ESR), rheumatoid factor and serum uric acid are within normal range within the subtype $[2,4]$.

Secondary subcategory of lipoma arborescens is designated as "synovial lipomatosis" and is associated with underlying, chronic irritation arising due to degenerative joint disease, trauma, meniscal injury or synovitis and is frequently discerned in elderly subjects $[2,4]$. 
Sub-synovial proliferation of adipose tissue is associated with three distinctive morphological patterns.

a) Diffuse villous arrangementincriminatinghypertrophied synovium in its entirety

b) Focal, nodular, frond-like configuration

c) Mixed articulation, arising due to an admixture of aforementioned patterns $[3,4]$.

\section{Histological elucidation}

On gross examination, lipoma arborescens typically demonstrates frond-like papillary excrescences. Macroscopically, yellowish, mature adipose tissue is delineated within villous, frond-like articulations. Of soft consistency, the mass is compressible [3,4]. On microscopy, villous configurations and synovial papillary proliferations are discerned along with substitution of sub-synovial tissue with mature adipocytes $[4,5]$.

The articular lesion comprises of sub-synovial, villouslike proliferation of mature adipose tissue cells. Hypertrophic villi are incorporated with significant accumulation of mature adipocytes and replace normal synovium $[4,5]$. Hypertrophic villous projections composed of mature adipose tissue are layered with synovial cells and a variable smattering of chronic inflammatory cells $[4,5]$.

Several instances are associated with diverse pathologies such as frequent knee joint effusion, commonly discerned degenerative modifications, lacerations of joint meniscus, infrequently observed synovial cysts, bone erosions or chondromatosis and the exceptional patellar subluxation or discoid meniscus $[4,5]$.

\section{Differential diagnosis}

Lipoma arborescens necessitates clinical segregation from categorical subgroups of articular masses as the noninfectious synovial proliferative lesions, infectious granulomatous conditions, depositional joint disease or articular masses of neoplastic and vascular origin. Commonly, synovial osteochondromatosis, pigmented villonodular synovitis (PVNS), rheumatoid arthritis, tuberculous arthritis or gouty arthropathy are conditions requiring differentiation. Aforesaid conditions display specific imaging features particularly upon magnetic resonance (MR) imaging [5,6].

Upon radiographic imaging, demarcation is required from pertinent conditions

- Loose bodies, frequently calcified, which appear hypointense upon magnetic resonance imaging (MRI) sequences $[5,6]$.

- Synovial osteochondromatosis or synovial chondromatosis exemplify circumscribed, occasionally ossified loose bodies along with foci of osseous erosion. Nod- ules of synovial chondromatosis may calcify. Synovial osteochondromatosis displays dispersion of typical cartilaginous or ossified loose bodies within the swollen joint $[6,7]$.

- Pigmented villonodular synovitis (PVNS) is a condition depicting firm, non-compressible nodules, as an adipose tissue constituent is absent. Upon radiography, bony sclerosis and erosions of articular or juxta-

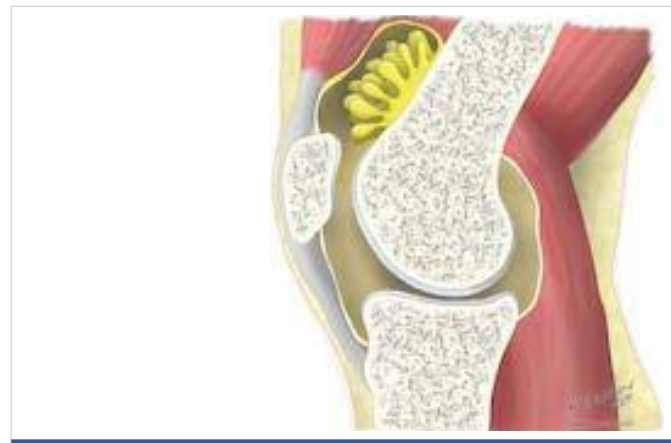

Figure 1: Lipoma arborescens depicting a supra-patellar aggregation of mature adipose tissue with frond-like, papillary projections.

Courtesy: Radiopedia.com.

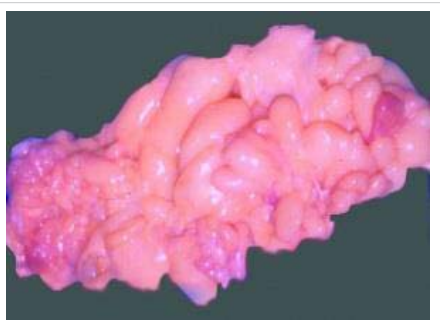

Figure 2: Lipoma arborescens demonstrating greasy, haemorrhagic, frond-like excrescences with an abundance of mature adipose tissue. Courtesy: Pathology outlines.

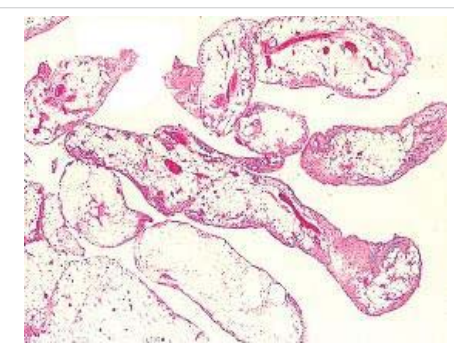

Figure 3: Lipoma arborescens enunciating papillary projections with a synovial epithelial lining, impaction with mature adipose tissue cells and red cell extravasation. Courtesy: Radiopedia.com

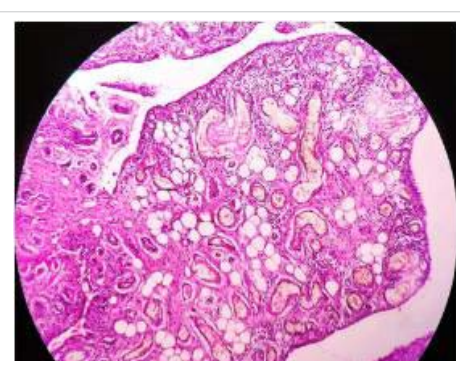

Figure 4: Lipoma arborescens exemplifying frond-like excrescences layered with synovial epithelium and imbued with mature adipose tissue cells. Courtesy: Medcrave.com 


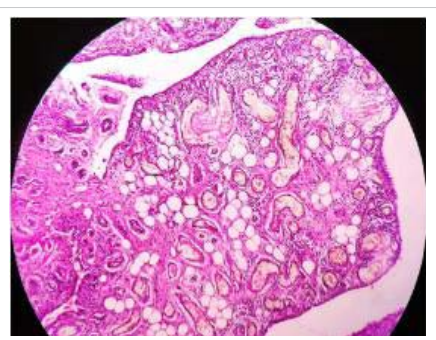

Figure 5: Lipoma arborescens exhibiting papillary projections coated with synovial tissue and impacted with mature adipose tissue cells.

Courtesy: Radsource.com

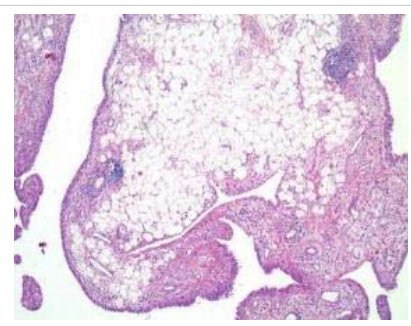

Figure 6: Lipoma arborescens delineating frond-like projections incorporated with mature adipose tissue cells and a superimposed layer of synovial epithelium. Courtesy: Springer link.com

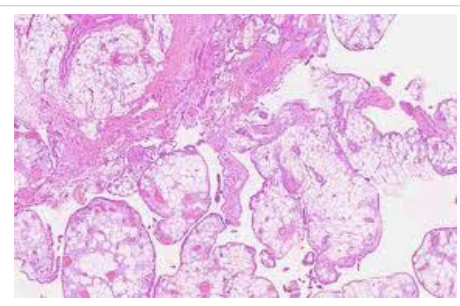

Figure 7: Lipoma arborescens depicting papillary projections incorporated with mature adipose tissue cells and a layering of synovial epithelium. Courtesy: Acta scientific.com

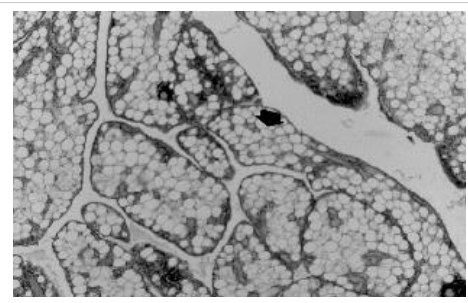

Figure 8: Lipoma arborescens demonstrating clusters of mature adipose tissue cells accumulated in the suprapatellar region, layered with synovial epithelium. Courtesy: Semantic scholar

articular surface is denominated. Upon computerized tomography (CT), enhanced attenuation of soft tissue is observed. Minimal signal intensity is detected upon T2 weighted imaging. Adipose tissue signal is absent [6,7].

Radiographic absence of bony erosion and sclerosis, firm consistency of periarticular soft tissue mass as discerned upon ultrasonography, enhanced density of internal contents, lack of erosion of articular surfaces upon computerized tomography (CT) and the absence of haemorrhagic elements or susceptibility signal upon magnetic resonance (MR) imaging eliminate the presence of pigmented villonodular synovitis $[7,8]$.
- Synovial haemangioma is associated with significant enhancement upon MRI with occasional demonstration of fluid- fluid levels.

- Synovitis delineates thickened synovium in the absence of an adipose tissue signal $[7,8]$.

- Gouty arthritis demonstrates typical juxta-articular, bony erosions, calcified soft tissue nodules or crystals within joint effusion.

- Tuberculous arthritis is an infective condition which is appropriately diagnosed upon clinical representation, pertinent imaging and biochemical parameters. Suspected instances can be subjected to an arthroscopic examination which can directly visualize the lesions, obtain cogent tissue samples and appropriately treat essential precipitating or coexistent conditions $[7,8]$.

\section{Investigative assay}

Aspiration of joint effusion demonstrates an absence of crystal deposition, cellular constituents and bacterial products $[7,8]$. Plain radiography can detect foci of enhanced, adipose tissue induced radiolucent shadows within a soft tissue lesion with predominant joint effusion. Concurrent degenerative alterations are frequent whereas erosion of osseous tissue is uncommon. Plain radiography provides limited benefit in discerning lipoma arborescens. Foci of radiolucency are infrequently observed within periarticular soft tissue along with nodular opacities which represents concomitant, prominent, adipose tissue projections [8,9]. Majority of incriminated knee joints are associated with degenerative modifications. Secondary radiographic manifestations demonstrate nonspecific fullness and soft tissue opacity within the suprapatellar pouch. Absence of bony sclerosis as well as articular and juxta-articular erosions of articular surface is discerned, in contrast to gouty arthropathy $[8,9]$. Ultrasonography of lipoma arborescens displays adipose tissue-rich villous projections with a typically enhanced echopattern simulating adjacent subcutaneous adipose tissue accompanied with real time undulations as the surrounding joint effusion. Ultrasonography is inexpensive, convenient and precise in localizing and determining extent of joint involvement with lipoma arborescens in diverse synovial surfaces. The procedure can be appropriately employed for initial diagnosis with subsequent adoption of cross sectional imaging $[8,9]$.

Ultrasonography demonstrates joint effusion and is associated with echogenic, frond- like synovial projections within the effusion $[9,10]$.

Computerized tomography (CT) delineates an intraarticular mass of minimal density. Density of joint effusion fluid is between adipose tissue and water and appears unamenable to radiographic enhancement with contrast administration $[9,10]$. 
Computerized tomography (CT) is exceptionally employed for evaluating lipoma arborescens. Scans demonstrate characteristic adipose tissue-rich synovial villi or frond- like projections interspersed within the circumscribing soft tissue associated with synovial thickening and joint effusion $[9,10]$.

Akin to adipose tissue -rich conditions, lipoma arborescens generally displays a specific signal upon magnetic resonance (MR) imaging which distinguishes the condition from associated intra-articular lesions. Therefore, MRI is a preferred investigative modality to evaluate the condition $[10,11]$.

Lipoma arborescens displays enhanced signal intensity of villous or nodular foci with T1 and T2 weighted imaging. Signal intensity is suppressed upon short tau inversion recovery (STIR) or fat- saturation sequences, recapitulating normal subcutaneous adipose tissue. Non adipose tissue component of hypertrophied synovium delineates heterogeneous, enhanced signal intensity upon T2 weighted imaging or STIR sequences and intermediate to minimal signal intensity upon T1 weighted imaging. Upon contrast administration, hypertrophied sub-synovial adipose tissue with superimposed thickened synovium is diffusely enhanced $[10,11]$.

MRI can also detect associated abnormalities such as joint effusion which manifest at initial representation. Secondary lipoma arborescens is accompanied by degenerative alterations and meniscal lacerations, in contrast to the primary variant $[10,11]$. As, magnetic resonance imaging (MRI) is the optimal investigative modality, it is typically associated with an appearance of adipose tissue -rich, frond- like, synovial mass with concurrent joint effusion. Upon T1 and T2 weighted imaging, the lesion denominates enhanced signal intensity, in association with saturated adipose tissue suppressed sequences. Gradient echo (GE) is occasionally associated with chemical shift artefact upon fat- fluid interface $[10,11]$.

Therapeutic Options Lipoma arborescens is a benign, indolent condition which can be managed conservatively. Symptomatic instances require aggressive surgical intervention. The essentially benign condition is appropriately alleviated by synovectomy $[10,11]$.

Primary therapeutic strategy for managing lipoma arborescens mandates mitigation of concurrent precipitating conditions. Aforesaid manoeuvers decimate the progression of secondary lipoma arborescens and associated clinical symptoms [10,11]. Advanced primary and challenging secondary instances of lipoma arborescens can be subjected to surgical intervention. Optimal surgical therapy is open or arthroscopic synovectomy. Arthroscopic synovial resection is minimally invasive with rapid, postoperative alleviation of cogent clinical symptoms $[10,11]$.

Posited as a reactive process, the lesion may be associated with reoccurrence. Lesion reoccurrence following competent employment of surgical synovectomy is uncommon.
Exceptional instances of extra-articular lipoma arborescens can be appropriately managed with open surgical excision, a technique which is applicable to adjunctive benign, periarticular masses $[10,11]$.

\section{Summary}

Lipoma arborescens is an exceptional condition comprised of frond-like excrescences of mature adipose tissue. The condition is posited to be a nonspecific, reactive proliferation of synovial adipose tissue arising secondary to chronic trauma or an inflammatory stimulus. Commonly, joint swelling with frequent joint effusion and an arthralgia of varying duration is exemplified. On microscopy, villous configurations and synovial papillary proliferations are discerned along with substitution of sub-synovial tissue with mature adipocytes.

Lipoma arborescens necessitates clinical segregation from articular masses as the noninfectious synovial proliferative lesions, infectious granulomatous conditions, depositional joint disease or articular masses of neoplastic and vascular origin. Commonly, synovial osteochondromatosis, pigmented villonodularsynovitis(PVNS), rheumatoidarthritis,tuberculous arthritis, gouty arthropathy, synovial haemangioma or loose bodies require a demarcation. Ultrasonography is inexpensive, convenient and precise in localizing the lesion and determining extent of joint involvement. Lipoma arborescens is a benign, indolent condition which can be managed conservatively although symptomatic instances require aggressive surgical intervention with procedures such as synovectomy.

\section{References}

1. Hoffa A. The influence of adipose tissue with regard to the pathology of the knee joint. J Am Med Association. 1904; 43: 795-796.

2. Arzimanoglu A. Bilateral arborescent lipoma of the knee. J Bone Joint Surg Am. 1957; 39: 976-979.

PubMed: https://pubmed.ncbi.nlm.nih.gov/13438955/

3. Schubert T, Navez M, Galant C, Docquier PL, Acid S, et al. Femoral osteochondroma responsible for ischiofemoral impingement, bursitis and secondary lipoma arborescens mimicking malignant transformation. Acta Radiol Open. 2019; 8: 2058460119892409.

PubMed: https://pubmed.ncbi.nlm.nih.gov/31903223/

4. Sanamandra SK, Ong KO. Lipoma arborescens. Singapore Med J. 2014; 55: 5-10.

PubMed: https://pubmed.ncbi.nlm.nih.gov/24452971/

5. Theermann R, Ohlmeier M, Hartwig CH, Wolff M, Krenn V, et al. Lipoma arborescens - Uncommon Diagnosis for Joint Swelling: Case Report and Review of the Literature. Z Orthop Unfall. 2020; 158: 618-624. PubMed: https://pubmed.ncbi.nlm.nih.gov/31746441/

6. Batu ED, Sonmez HE, et al. Lipoma Arborescens Associated With Psoriatic Arthritis in an Adolescent Boy: A Case Report and Review of the Literature. J Clin Rheumatol. 2020; 26: e47-e49. PubMed: https://pubmed.ncbi.nlm.nih.gov/31746441/

7. Kord Valeshabad A, De La Vara D, Shamim E, Alsadi A, et al. Lipoma arborescens of the bicipitoradial bursa. Skeletal Radiol. 2018; 47: 549-551. PubMed: https://pubmed.ncbi.nlm.nih.gov/29079872/

8. Howe BM, Wenger DE. Lipoma arborescens -comparison of typical 
and atypical disease presentation. Clin Radiol. 2013; 68: 1220-1226. PubMed: https://pubmed.ncbi.nlm.nih.gov/23969149/

9. Hamanishi M, Yasunaga Y, Yamasaki T, Mori R, Shoji T, et al. Lipoma arborescens of the hip-a case report. J Orthop Sci. 2020; 25: 188-192. PubMed: https://pubmed.ncbi.nlm.nih.gov/28688812/

10. Kim RS, Kim YT, Choi JM, Shin SH, Kim YJ, et al. Lipoma arborescens associated with osseous /chondroid differentiation in subdeltoid burs.
Int J Shoulder Surg. 2013; 7: 116-119.

PubMed: https://www.ncbi.nlm.nih.gov/pmc/articles/PMC3807946/

11. Natera L, Gelber PE, Erquicia JI, Monllau JC. Primary lipoma arborescens of the knee may involve the development of early osteoarthritis if prompt synovectomy is not performed. J Orthop Traumatol. 2015; 16: 47-53.

PubMed: https://pubmed.ncbi.nlm.nih.gov/24796311/ 